\title{
EDITORIAL
}

\section{Is it really time to look at distal airways to improve asthma phenotyping and treatment?}

\author{
T. Perez
}

I n 2011, severe asthma and/or insufficient control of disease remain major challenges for the pulmonologist [1]. Small airway involvement has been demonstrated by a number of studies, particularly in fatal, severe or poorly controlled asthma, and may be a potential target to improve treatment and asthma outcomes [2-5]. Although inhaled corticosteroids (ICSs) remain the cornerstone of asthma treatment, there is a continuing debate on the potential benefit of small-particle formulations. In this context, the recent study by COHEN et al. [6] clearly adds new data to this controversial topic.

It is the first to compare airway hyperresponsiveness (AHR) to small-particle adenosine monophosphate (AMP) with large and standard particles in a reasonable sample of mild-to-moderate asthmatics. In addition, the impact of small- (ciclesonide) versus large-particle (fluticasone) ICS formulations was compared at 4 weeks using the same original AMP challenges, exhaled NO and spirometry. Unfortunately, asthma control was not assessed. The results clearly indicate that small-particle ICSs may improve corresponding AHR, but other functional outcomes were surprisingly not correlated with this benefit. Such an approach targeting evaluation and treatment of small airways raises a series of questions.

AHR is a complex phenomenon in asthma and the contribution of small airways to this hyperresponsiveness is poorly characterised, particularly regarding its variable inflammatory component. Distal airway inflammation has been demonstrated by a number of histopathological studies using lung resection specimens or transbronchial biopsies [2]. Taken together, these data suggest an important role for distal airway inflammation in severe asthma.

Consistent with these histopathological findings, direct measurement of distal airway resistance showed that they accounted for up to 50-90\% of total airflow resistance in asthma [7], implying that distal airways were the main site of airflow obstruction. Early experimental and theoretical models suggested that small airways were also the predominant determinants of increased airway resistance, explaining the lack of plateau in acute bronchoconstriction, for instance, during AHR testing [8]. In addition, peripheral airway responsiveness (by direct measurement through a fibreoptic catheter) induced by histamine challenge was significantly enhanced in asthmatic subjects relative to normal controls [9].

CORRESPONDENCE: T. Perez, Dept of Respiratory Medicine and Lung Function Dept, Lille University Hospital, 59037 Lille, France. E-mail thierry.perez@chru-lille.fr
Contrary to what was expected according to these classical data, small-particle provocative concentration causing a $20 \%$ fall in forced expiratory volume in $1 \mathrm{~s}$ (PC20) was much higher than large-particle PC20 in the study by COHEN et al [6], which might be due either to exceedingly small AMP particle size (mass median aerodynamic diameter (MMAD) $1.04 \mu \mathrm{m}$ ) or, more likely, to an inadequacy of forced expiratory volume in $1 \mathrm{~s}$ (FEV1) to measure small airway bronchoconstriction. Indeed, using low-frequency forced oscillation resistance, SEKIZAWA et al. [10] demonstrated a marked increase in small airway resistance in eight out of 13 asthmatics during a methacholine challenge using standard-sized particles. The level of AHR was also more severe in this subset of patients.

To date, no data were available before those of COHEN et al. [6] regarding small airway AHR to an indirect stimulus, but their contribution has also been detected by high-resolution computed tomography (HRCT) and nitrogen washout during the late phase after cat allergen challenge [11].

Due to the well-demonstrated relationship between baseline reduction of airway calibre (either due to remodelling or active inflammatory changes) and the severity of AHR, the level of fine-particle AHR should also be linked to a reduced calibre of the small airways. This mechanism is confirmed in the study by COHEN et al. [6], with significantly lower forced expiratory flow at $25-75 \%$ of forced vital capacity (FEF25-75\%) in patients demonstrating positive small-particle AHR.

The second unexpected result of the study is that despite an improvement in small-particle AMP PC20 with ciclesonide, two other indexes of small airways dysfunction, FEF25-75\% and alveolar exhaled NO, improved more significantly in the fluticasone arm. The reasons for such discrepancy are not entirely clear; the authors postulate an insufficient duration of treatment (only 4 weeks), an influence of pre-treatment by another ICS, or a dosage difference between the ciclesonide and fluticasone arms. Alternatively, FEF25-75\% also has several limitations and may not be the optimal tool for such assessments [12, 13].

Other recent studies focusing on small airways using various methods demonstrated a significant effect of small-particle ICSs at this level. Ciclesonide had a significant impact on small airway function, as demonstrated by forced vital capacity change during AHR, HRCT air trapping and exhaled alveolar $\mathrm{NO}$ in a small sample of patients [14]. Regarding remodelling and inflammatory changes, flunisolide hydrofluoroalkane (HFA), another fine-particle ICS, significantly decreased bronchiolar smooth muscle actin area without any change in 
collagen content [15]. In addition, a reduction of eosinophils in late-phase induced sputum was observed with ciclesonide [16], in parallel with an improvement in oscillatory parameters reflecting small airways function.

However, the overall prevalence and clinical relevance of distal airway dysfunction remains difficult to establish in the general population of asthmatic patients, most available studies having focused on small numbers of poorly controlled or severe disease. The main difficulty is the lack of reliable and widely available lung function parameters able to detect distal airways involvement in clinical practice. A number of parameters have been proposed but most techniques, and particularly the most specific, remain confined to highly specialised lung function laboratories and none can be considered, at present, as the gold standard [3-5].

The single-breath washout has only recently been re-evaluated in asthma. BOURDIN et al. [17] showed that poor asthma control correlated with an increase in closing volume and phase-III slope. Other investigators reported that increased closing volume correlated with frequent asthma exacerbations and residual volume/total lung capacity ratio [18]. The impact of therapy on these parameters remains currently unknown.

VERBANCK et al. [19] described an exclusive method of multiplebreath nitrogen washout (MBW) analysis, defining two new variables, Scond (index of conductive ventilation) and Sacin (index of acinar ventilation), as measurements of ventilation inhomogeneity in their respective zones. In asthma, the most consistent pattern of non- $\beta_{2}$-agonist-reversible ventilatory heterogeneity was found in the conductive lung zone, most probably in the small conductive airways [20]. Scond appears strongly correlated to the level of direct AHR (methacholine) in patients treated with ICS [21]. These investigators further showed that only those with baseline acinar lung zone abnormality (Sacin) obtained a functional benefit on small airways with HFA-beclomethasone, another extra-fine ICS [22].

The contribution of distal airways to exhaled NO may be evaluated by alveolar NO (Calv,NO), which was shown to increase in severe asthmatics in parallel with bronchoalveolar lavage eosinophils [23]. Calv,NO was found to correlate with distal airway obstruction, as measured by spirometry [24] and single-breath nitrogen test [25]. Calv,NO was also highly correlated with Sacin index in ICS-treated patients [26]. The impact of fine-particle ICSs on alveolar NO was markedly different in the two studies by COHEN and co-workers, as it was positive in one [14], but nonsignificant in the latter [6].

Forced oscillation and impulse oscillometry are simple noninvasive techniques, although not widely available at present. A predominant increase of the resistance of the respiratory system (Rrs) at low frequencies (less than 10-15 Hz), called frequency dependence of resistance, has been shown to reflect obstruction in distal airways. Low-frequency reactance may also evaluate small-airway compliance [27]. Increased low-frequency $R$ rs values were found in asthmatic children with mild airway obstruction (FEV1 $>80 \%$ predicted and $\mathrm{FEF} 25-75 \%<80 \%$ pred) [28]. In adults, subjects with moderate and severe asthma also exhibited a marked frequency dependence of $\operatorname{Rrs}$ [29]. Impulse oscillometry was able to demonstrate significant changes in lowfrequency parameters after 8-12 weeks of small-particle ICS treatment in two concordant studies [13, 16]. In the latter, oscillometry parameters improved despite nonsignificant changes in spirometry indices, including FEF25-75\%.

A significant limitation of the study by COHEN et al. [6] is that both proximal and distal AHR were evaluated with FEV1 and interrupter resistance measurements, both poor indices of distal airway physiology. Other indices described above should have preferably been used to evaluate small-airway AHR and the impact of small- versus large-particle ICSs on these airways.

Determining the predominant site of indirect AHR in an individual patient with the tools used by COHEN et al. [6] is complex, requiring specific nebulisers with an optical control of MMAD, particularly for the small-size challenge. MBW Scond-Sacin and this specific AHR testing of small airways are promising research tools but are unlikely to become standard procedures. In addition, modulation of asthma treatment by monitoring AHR is not recommended at present, and generally leads to increased cumulative ICS doses. Whether fine-particle AHR provocation could constitute a relevant criteria for the long-term follow-up of patients with small airway involvement remains to be determined.

Altogether, these recent data suggest that a fine tuning of ICS treatment may significantly improve different sites of AHR and airway inflammation, which could, in theory, translate into better asthma control and long-term outcome. Large clinical trials only showed a noninferiority of fine-particle ICS despite lower dosage, not a clear superiority $[3,4,30]$. However, none of these trials selected patients with baseline distal airway involvement, in whom the effects of a targeted small-particle treatment could be potentially larger.

The findings of recent studies focusing on small airways should, of course, be confirmed by large-scale trials, in patients with different patterns of severity and control. Actually, such an approach is clearly limited by the insufficient availability and relative complexity of small-airway evaluation techniques. In this context, impulse oscillometry might be a reasonable candidate to evaluate larger patient populations in the next future.

For the pulmonologist, the practical questions in 2011 are still as follows. 1) Should small airways be systematically assessed in poorly controlled or severe asthma and, if the answer is yes, by which clinically relevant and widely available method? 2) In patients with demonstrated small-airway dysfunction ("small airway phenotype"), what is the additional benefit of smallparticle ICS (and new drugs targeting distal airways) or systemic treatments on specific parameters and usual clinical outcomes?

\section{STATEMENT OF INTEREST}

A statement of interest for T. Perez can be found at www.erj. ersjournals.com/site/misc/statements.xhtml

\section{REFERENCES}

1 Taylor DR, Bateman ED, Boulet LP, et al. A new perspective on concepts of asthma severity and control. Eur Respir J 2008; 32: 545-554.

2 Kraft M. The distal airways: are they important in asthma? Eur Respir J 1999; 14: 1403-1417. 
3 Contoli M, Bousquet J, Fabbri LM, et al. The small airways and distal lung compartment in asthma and COPD: a time for reappraisal. Allergy 2010; 65: 141-151.

4 Van den Berge M, ten Hacken NH, Cohen J, et al. Small airway disease in asthma and COPD: clinical implications. Chest 2011; 139: 412-423.

5 Burgel PR, de Blic J, Chanez P, et al. Update on the roles of distal airways in asthma. Eur Respir Rev 2009; 18: 80-95.

6 Cohen J, Postma DS, Douma WR, et al. Particle size matters: diagnostics and treatment of small airways involvement in asthma. Eur Respir J 2011; 37: 532-540.

7 Yanai M, Sekizawa K, Ohrui T, et al. Site of airway obstruction in pulmonary disease: direct measurement of intrabronchial pressure. J Appl Physiol 1992; 72: 1016-1023.

8 Wiggs BR, Bosken C, Pare PD, et al. A model of airway narrowing in asthma and in chronic obstructive pulmonary disease. Am Rev Respir Dis 1992; 145: 1251-1258.

9 Wagner EM, Bleecker ER, Permutt S, et al. Direct assessment of small airways reactivity in human subjects. Am J Respir Crit Care Med 1998; 157: 447-452.

10 Sekizawa K, Sasaki H, Shimizu Y, et al. Dose-response effects of methacholine in normal and in asthmatic subjects. Relationship between the site of airway response and overall airway hyperresponsiveness. Am Rev Respir Dis 1986; 133: 593-599.

11 Zeidler MR, Goldin JG, Kleerup EC, et al. Small airways response to naturalistic cat allergen exposure in subjects with asthma. J Allergy Clin Immunol 2006; 118: 1075-1081.

12 Boulet LP. Comparative improvement of asthma symptoms and expiratory flows after corticosteroid treatment: a method to assess the effect of corticosteroids on large $v$ s. small airways? Respir Med 2006; 100: 496-502.

13 Yamaguchi M, Niimi $A$, Ueda $T$, et al. Effect of inhaled corticosteroids on small airways in asthma: investigation using impulse oscillometry. Pulm Pharmacol Ther 2009; 22: 326-332.

14 Cohen J, Douma WR, ten Hacken NH, et al. Ciclesonide improves measures of small airway involvement in asthma. Eur Respir J 2008; 31: 1213-1220.

15 Bergeron C, Hauber HP, Gotfried M, et al. Evidence of remodeling in peripheral airways of patients with mild to moderate asthma: effect of hydrofluoroalkane-flunisolide. J Allergy Clin Immunol 2005; 116: 983-989.

16 Hoshino M. Comparison of effectiveness in ciclesonide, fluticasone propionate on small airway function in mild asthma. Allergol Int 59: 59-66.
17 Bourdin A, Paganin F, Prefaut C, et al. Nitrogen washout slope in poorly controlled asthma. Allergy 2006; 61: 85-89.

18 van Veen $\mathrm{IH}$, Sterk PJ, Schot $\mathrm{R}$, et al. Alveolar nitric oxide versus measures of peripheral airway dysfunction in severe asthma. Eur Respir J 2006; 27: 951-956.

19 Verbanck S, Schuermans D, Van Muylem A, et al. Ventilation distribution during histamine provocation. J Appl Physiol 1997; 83: 1907-1916.

20 Verbanck S, Schuermans D, Paiva M, et al. Nonreversible conductive airway ventilation heterogeneity in mild asthma. J Appl Physiol 2003; 94: 1380-1386.

21 Downie SR, Salome CM, Verbanck S, et al. Ventilation heterogeneity is a major determinant of airway hyperresponsiveness in asthma, independent of airway inflammation. Thorax 2007; 62: 684-689.

22 Verbanck S, Schuermans D, Paiva M, et al. The functional benefit of anti-inflammatory aerosols in the lung periphery. J Allergy Clin Immunol 2006; 118: 340-346.

23 Berry M, Hargadon B, Morgan A, et al. Alveolar nitric oxide in adults with asthma: evidence of distal lung inflammation in refractory asthma. Eur Respir J 2005; 25: 986-991.

24 Mahut B, Delacourt C, Zerah-Lancner F, et al. Increase in alveolar nitric oxide in the presence of symptoms in childhood asthma. Chest 2004; 125: 1012-1018.

25 Battaglia S, den Hertog H, Timmers MC, et al. Small airways function and molecular markers in exhaled air in mild asthma. Thorax 2005; 60: 639-644.

26 Verbanck S, Schuermans D, Vincken W. Inflammation and airway function in the lung periphery of patients with stable asthma. J Allergy Clin Immunol 2010; 125: 611-616.

27 Goldman MD, Saadeh C, Ross D. Clinical applications of forced oscillation to assess peripheral airway function. Respir Physiol Neurobiol 2005; 148: 179-194.

28 Delacourt C, Lorino $\mathrm{H}$, Herve-Guillot $\mathrm{M}$, et al. Use of the forced oscillation technique to assess airway obstruction and reversibility in children. Am J Respir Crit Care Med 2000; 161: 730-736.

29 Cavalcanti JV, Lopes AJ, Jansen JM, et al. Detection of changes in respiratory mechanics due to increasing degrees of airway obstruction in asthma by the forced oscillation technique. Respir Med 2006; 100: 2207-2219.

30 Gentile DA, Skoner DP. New asthma drugs: small molecule inhaled corticosteroids. Curr Opin Pharmacol 2010; 10: 260-265. 